\title{
BIM-Sim: Interactive Simulation of Broadband Imaging Using Mie Theory.
}

\author{
Sebastian Berisha ${ }^{1}$, Thomas van Dijk ${ }^{2}$, Rohit Bhargava ${ }^{3}$, P. Scott Carney ${ }^{3}$, and David Mayerich ${ }^{1}$ \\ 1. University of Houston, Department of Electrical and Computer Engineering, Houston, Texas. \\ 2. Department of Medical Physics, Máxima Medical Centre, Veldhoven, The Netherlands. \\ 3. University of Illinois at Urbana-Champaign, Department of Electrical and Computer Engineering, \\ Urbana-Champaign, Illinois.
}

Mie theory plays an important role in describing the scattering of electromagnetic (EM) fields by providing a rigorous solution to Maxwell's Equations for a spherical scatterer based on an incident plane wave. This theory has been used to characterize absorption in atmospheric simulations [1], and to approximate cellular structures in spectroscopic imaging of biological tissues [2]. There is also interest in using micro and nanospheres, such as quantum dots, to improve sensing in biomedicine [3].

The problem of inverse scattering in samples composed of particulate or spherical components is of particular interest. In our previous work [4], we demonstrated a method for computing the refractive indices of spheres composed of polymers measured using mid-infrared point spectroscopy. However, these nonlinear solutions require iterative computation of the forward model. Since Mie theory is computationally expensive, this solution becomes impractical for multidimensional spectroscopic images. Studying the behavior of EM fields generates a time-dependent component, which requires a 5D simulation in $(x, y, z, \lambda, t)$ that is both computationally expensive and requires a prohibitive amount of memory.

We have developed a software package called the Broadband Interactive Mie Simulator (BIM-Sim), which interactively computes the scattered fields produced by spherical particles. This includes the simulation of the entire imaging system, including focusing and imaging optics as well as a detector. The complete field is computed both inside and outside of each particle. We use a mathematical model based on Mie theory that allows efficient and highly-parallel evaluation of the scattered field. The highdimensional nature of the simulation is constrained by user-specified visualization parameters. By taking advantage of GPU-based computation, we allow the user to interactively adjust these parameters to perform the simulation in real-time.

We visualize the field using planar cross-sections of the volume placed anywhere between the objective and condenser. The complex components of the scattered field at the particle surface are computed and visualized using a 3D surface model. This facilitates the study of the scattering efficiency of a sphere in three dimensions. The shape of the scattered field is computed using Monte-Carlo sampling, which allows arbitrary positioning of the particle within the focal volume.

The imaging process is simulated by band-limiting a cross-section of the field at the focal plane based on the objective aperture function. The intensity of the filtered cross-section is computed and re-sampled based on user-specified detector parameters to create a final image (Figure 1). Computing both the total and incident field intensity also allows the simulation of absorption spectroscopy for any distribution of micro-spheres. Material properties for the spheres are specified at run-time or as a wavelength dependent set of refractive indices. 
All of our results were tested on an NVIDIA GeForce GTX 970 with 4GB of memory and 13 multiprocessors. For a 256×256 resolution slice, full evaluation of the incident field (in CUDA) requires $4-5 \mathrm{~ms}$ and evaluation of the internal and scattered fields requires 175-192ms. This includes both computation of the scatter domain and 400 Monte-Carlo samples of a single sphere positioned in the near-field. Evaluation time scales linearly with the number of particles. Our current simulations are bounded by the amount of time required to fetch multiple samples of the scattered domain for MonteCarlo sampling. We have also implemented a MATLAB GPU-based version of BIM-Sim. As expected, the computational speed decreases compared to the CUDA implementation. In particular, for the same slice resolution, in MATLAB, it requires $220-270 \mathrm{~ms}$ for a full evaluation of the incident field and $4-5 \mathrm{~s}$ for the evaluation of the internal and scattered fields. We have found no other interactive tools for exploring the multidimensional scattered field produced by spheres.

BIM-Sim addresses the need for interactive simulation and visualization of multidimensional scattered EM fields. This is an important problem in the optics community, where there is significant interest in understanding how micro and nano-particles interact with incident radiation. Our two major contributions include a method for interactive simulation of scattered fields and a framework for visualizing these fields by coupling user interaction with sparse simulation.

\section{References:}

[1] T. C. Bond, G. Habib, and R. W. Journal of Geophysical Research: Atmospheres, 111(D20):D20211, Oct. 2006.

[2] P. Bassan, et al, Journal of Biophotonics, 3(8-9):609- 620, Aug. 2010.

[3] J. L. West and N. J. Halas. Annual Review of Biomedical Engineering, 5(1):285-292, 2003.

[4] T. van Dijk, et al, Applied Spectroscopy, 67(5):546-552, May 2013.

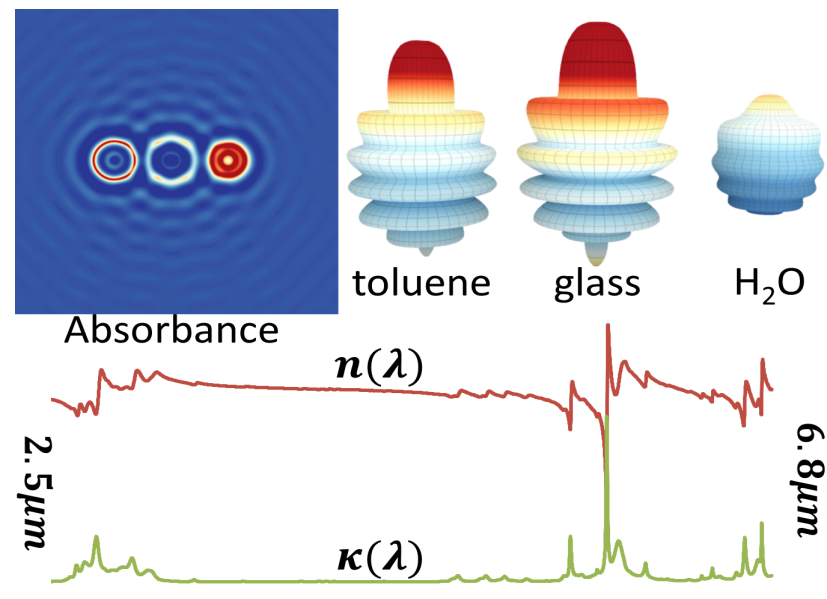

(a) $\eta(\lambda)$ for toluene, image at $\lambda=3.1 \mu \mathrm{m}$

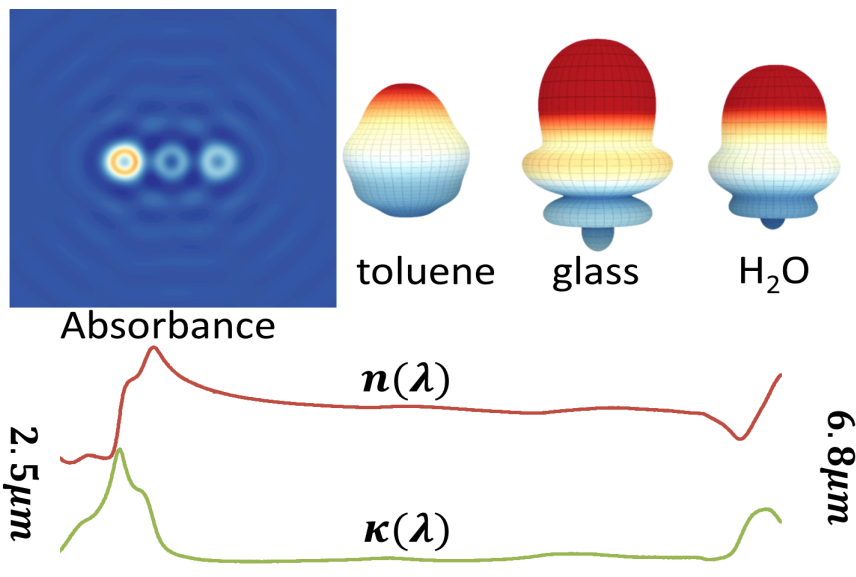

(b) $\eta(\lambda)$ for $\mathrm{H}_{2} \mathrm{O}$, image at $\lambda=6.6 \mu \mathrm{m}$

Figure 1. Absorption image and scattering efficiency of three spheres made of different materials with absorption spectra in the mid-infrared. (a) The absorption image (left) and scattering efficiency (right) is shown at $\lambda=3.1 \mu \mathrm{m}$. The real and imaginary parts of the index of refraction are shown for toluene. (b) The absorption image and scattering efficiency for $\lambda=6.6 \mu \mathrm{m}$ is shown (top) with the index of refraction for water $\left(\mathrm{H}_{2} \mathrm{O}\right.$, bottom $)$. 